\title{
A novel humanoid-robot simulator for colonoscopy
}

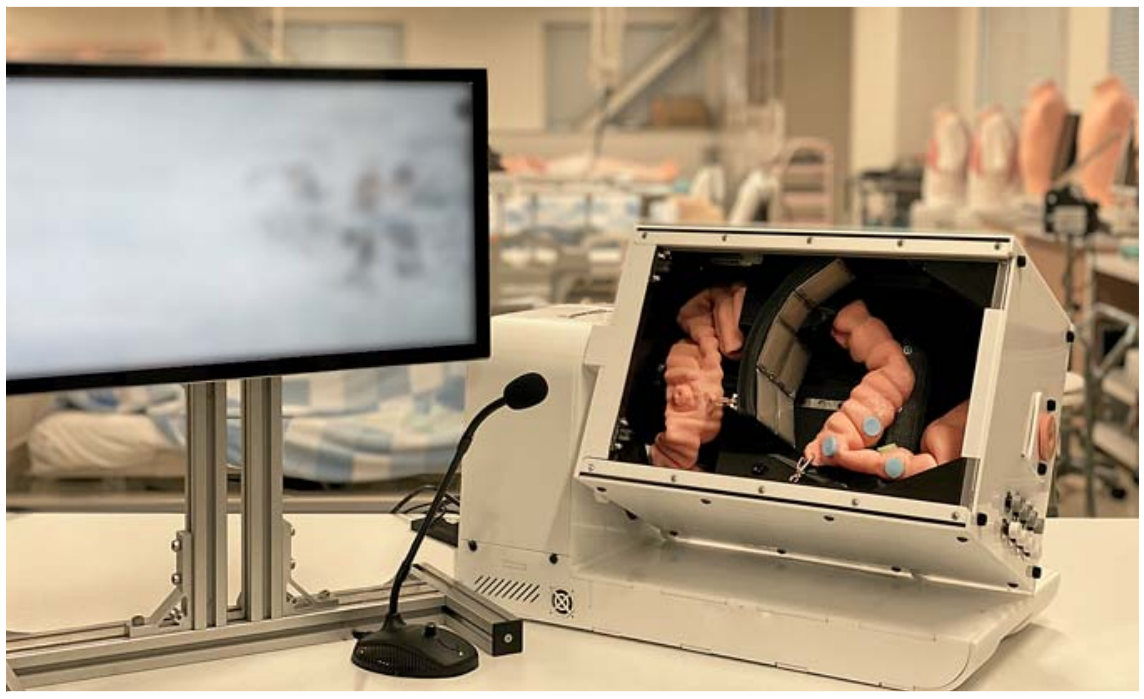

- Fig. 1 External view of colon simulator. The three-dimensional colon model is made of silicon resin. The humanoid-robot simulator has the ability to change body position.

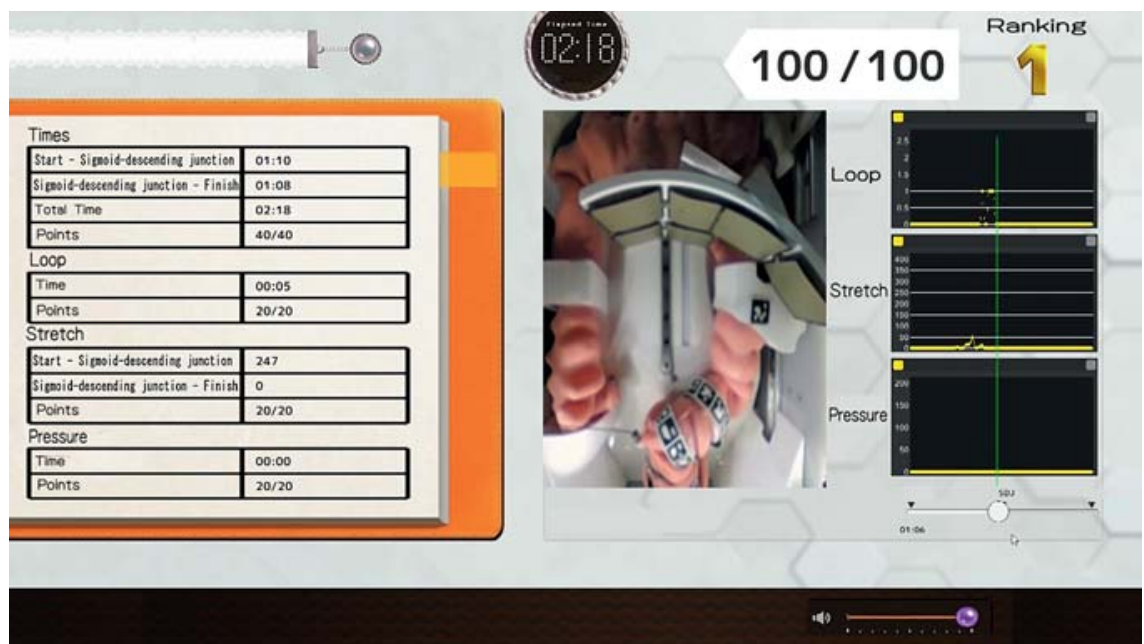

- Fig. 2 Screen layout for the evaluation of the simulation performed. During insertion of the colonoscope from anus to cecum, pain caused by the colonoscopy is evaluated by measuring the pressure exerted on the colon by the colonoscope and the distensibility of the colon using multiple sensors, such as a pressure sensor and an optical sensor. The humanoid-robot simulator can score the insertion of the colonoscope by evaluating the pressure on the colon from the colonoscope, the distensibility of the colon, and the duration of the procedure.

Colorectal cancer is the cancer with the third-highest incidence worldwide [1]. Colonoscopy is a useful imaging modality that has a high sensitivity and can reduce mortality from colorectal cancer [2]. Although diminutive adenomas pose a low- er risk of malignancy than larger ones, an increase in adenoma detection rates may contribute to decreasing the risk of colorectal cancer [3]. However, operator proficiency has been a difficult hurdle to overcome. Basic insertion skills are re-

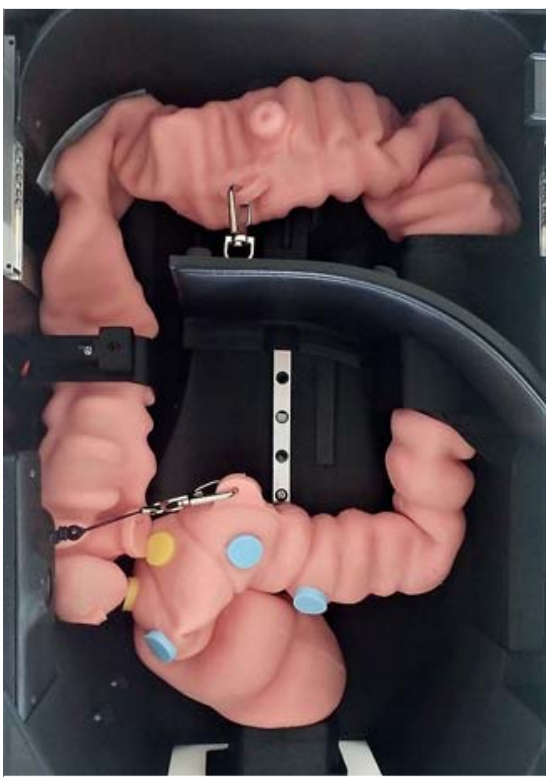

Fig. 3 External view of the silicon model of the colon. The prototype three-dimensional colon model was prepared using $\mathrm{CT}$ images of the human colon. The humanoid-robot simulator has several supporting functions, such as abdominal compression and repositioning of the diaphragm as if through deep inspiration.

quired to perform a standardized colonoscopy. As observing the whole lumen with as few blind spots as possible is a basic prerequisite for qualified endoscopic diagnosis, subjective factors or limited levels of ability in the endoscopists may lead to missed lesions. Endoscopic training is expensive in both time and costs, and there is also a lack of realistic simulators for trainees. For these reasons, we developed a humanoid-robot simulator, called a "mikoto," that allows endoscopists to insert the scope in a way that feels comparable to performing a colonoscopy in the human body ( $\bullet$ Fig. 1 ).

The three-dimensional colon model, the data for which was prepared using CT images of the human colon, is made of a silicon resin that is thinner and more flexible than conventional models ( $\bullet$ Fig. 2 , - Fig. 3, - Fig.4, > Fig.5). Insertion of the colonoscope can be scored by evalu- 


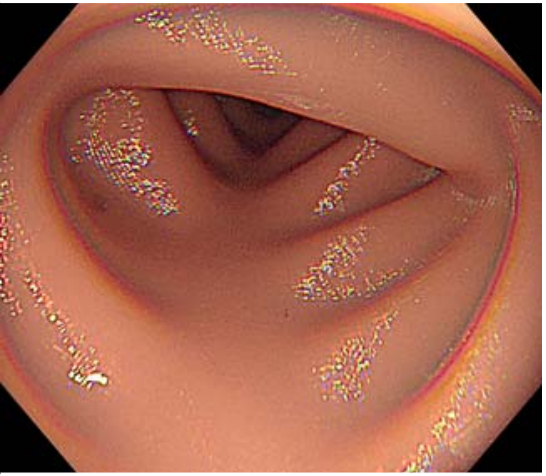

- Fig. 4 Lumen image of the silicon model. The three-dimensional colon model is constructed of silicon resin, which is thin and flexible. When endoscopists insert the endoscope from anus to cecum in the humanoid-robot simulator, it is similar to performing a colonoscopy in the human body.

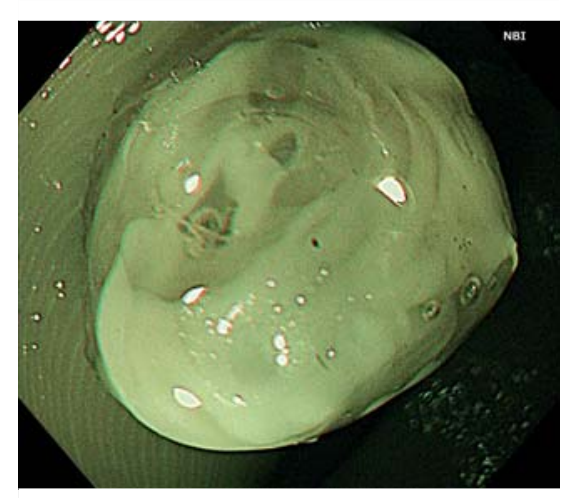

- Fig. 5 Image of a silicon model polyp with narrow-band imaging.

ating the pressure of the colonoscope on the colon, the distensibility of the colon, and the duration of the procedure ( Fig.2). The mikoto has additional functions, such as the ability to change the body position; the capability to adjust colonoscope insertion for different levels of difficulty, exert abdominal compression, and reposition the diaphragm as if through deep inspiration; and the ability to provide alerts when the colon forms a loop ( Video 1). The mikoto outperforms other self-training methods for trainees to learn how to insert the colonoscope successfully. Additionally, it is less invasive, and the performance scoring system provides more objective feedback than examinees might obtain in an actual clinical setting.

Endoscopy_UCTN_Code_TTT_1AQ_2AJ

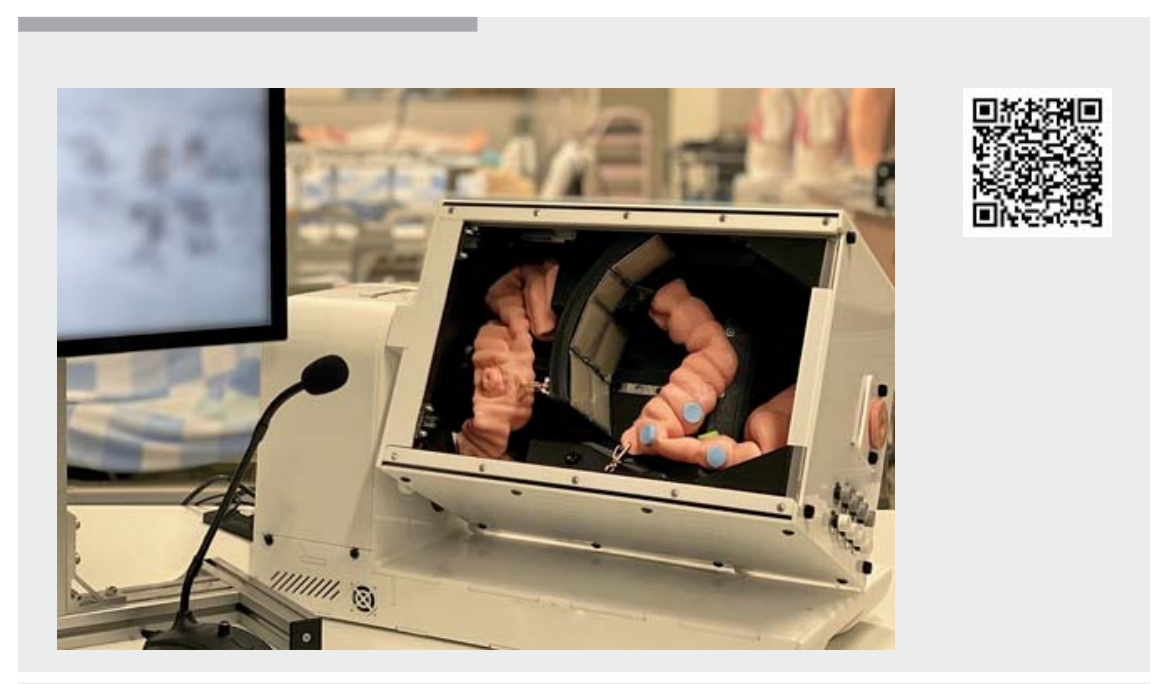

Video 1 A novel humanoid-robot simulator for colonoscopy.

\section{Competing interests}

Masashi Fujii was employed on an advisor of MICOTO Technology Inc. The other authors declare that they have no conflict of interest.

\section{The authors}

Masashi Fujii ${ }^{1,2}$, Takumi Onoyama1, Yuichiro Ikebuchi ${ }^{1}$, Kazutake Uehara ${ }^{2}$, Atsuro Koga ${ }^{3}$,

\section{Masaru Ueki ${ }^{4}$, Hajime Isomoto ${ }^{1}$}

1 Division of Gastroenterology and Nephrology, Department of

Multidisciplinary Internal Medicine, Tottori University Faculty of Medicine, Yonago, Japan

2 Advanced Medicine, Innovation and Clinical Research Center, Tottori University Hospital, Yonago, Japan

3 Research Strategy Division, Organization for Research Initiative and Promotion, Tottori University, Yonago, Japan

4 Division of Medical Education, Department of Medical Education, Tottori University Faculty of Medicine, Yonago, Japan

\section{Corresponding author}

\section{Takumi Onoyama, MD, PhD}

Division of Gastroenterology and Nephrology, Department of Multidisciplinary Internal Medicine, Tottori University Faculty of Medicine, 36-1 Nishi-cho, Yonago 6838504, Japan golf4to@yahoo.co.jp

\section{References}

[1] Ferlay J, Soerjomataram I, Dikshit R et al. Cancer incidence and mortality worldwide: sources, methods and major patterns in GLOBOCAN 2012. Int J Cancer 2015; 136 : E359-E386

[2] Lieberman DA. Clinical practice. Screening for colorectal cancer. N Engl J Med 2009; 361: 1179-1187

[3] Corley DA, Jensen CD, Marks AR et al. Adenoma detection rate and risk of colorectal cancer and death. N Engl J Med 2014; 370 : 1298-1306

[4] Ansell J, Mason J, Warren $\mathrm{N}$ et al. Systematic review of validity testing in colonoscopy simulation. Surg Endosc 2012; 26: 30403052

[5] Noda K, Kitada T, Suzuki Y et al. A novel physical colonoscopy simulator based on analysis of data from computed tomography colonography. Surg Today 2017; 47: 11531162

Bibliography

Endoscopy 2021; 53: E291-E292

DOI 10.1055/a-1264-6804

ISSN 0013-726X

published online 8.10 .2020

(c) 2020. Thieme. All rights reserved.

Georg Thieme Verlag KG, Rüdigerstraße 14,

70469 Stuttgart, Germany 